\title{
Higher Dimensional Integrable Models from Lower Ones via Miura Type Deformation Relation
}

\author{
Sen-yue Lou ${ }^{\mathrm{a}, \mathrm{b}}$, Jun $\mathrm{Yu}^{\mathrm{c}}$, and Xiao-yan Tang ${ }^{\mathrm{a}}$ \\ ${ }^{a}$ Applied Physics Department of Shanghai Jiao Tong University, Shanghai 200030, P. R. China \\ ${ }^{\mathrm{b}}$ Institute of Modern Physics, Ningbo University, Ningbo 315211, P. R. China \\ ${ }^{c}$ Department of Physics, Shaoxing College of Arts and Science, Shaoxing 312000, P. R. China
}

Reprint requests to Prof. S.-y. L.; E-mail: sylou@mail.sjtu.edu.cn

Z. Naturforsch. 55 a, 867-876 (2000); received August 15, 2000

\begin{abstract}
To find nontrivial high dimensional integrable models (especially in (3+1)-dimensions) is one of the most important problems in nonlinear physics. A systematic method to find some nontrivial high dimensional integrable models is established by means of noninvertible deformation relations. Starting from a noninvertible Miura type transformation, we find that the $(1+1)$-dimensional sinhGordon model appearing in many physical fields is a deformation of the (0+1)-dimensional Riccati equation. A high dimensional Miura type deformation (including two different (3+1)-dimensional reductions) of the heat conduction equation is proved to be Painlevé integrable. Some special types of explicit exact solutions, like multi-plane and/or multi-camber soliton solutions, multi-dromion solutions and multiple ring soliton solutions, are obtained.
\end{abstract}

Key words: Noninvertible Deformation; High Dimensional Integrable Models; Camber Solitons; Ring Solitons.

\section{Introduction}

Because of the wide application of soliton theory in almost every branch of physics, like field theory [1], condensed matter physics [2], fluid mechanics [3], plasma physics [4], optics [5] etc., various physicists and mathematicians have focused their attention on the study of integrable systems. However, because of the difficulty to find high dimensional integrable models, especially in $(3+1)$-dimensions, most of the studies on soliton systems are restricted in the lower dimensions ( $1+1$ and $2+1$ dimensions). Recently, one of the present authors (Lou) had pointed out some possible ways to get higher dimensional integrable models: (i) Based on the fact that all known (2+1)-dimensional integrable models possess a common generalized Virasoro type symmetry algebra [6],

$$
\left[\sigma\left(f_{1}\right), \sigma\left(f_{2}\right)\right]=\sigma\left(\dot{f}_{2} f_{1}-\dot{f}_{1} f_{2}\right),
$$

where $\sigma(f)$ with $f$ being an arbitrary function of a single independent variable, is a symmetry of the integrable models, we proposed a general method to get some integrable models in the sense that they possess the generalized Virasoro symmetry algebra (1) [7, 8]. (ii) Using a recursion operator of a $(1+1)$-dimensional integrable model, the breaking soliton [9] equation can be extended to any dimensions $[8,10]$. (iii) Using the inner parameter dependent symmetry constraints, one can also get some types of integrable models in the same or even higher dimensions [11]. (iv) Some quite general higher dimensional conformal invariant models are integrable, i.e. they possess the Painlevé property [12]. (v) In [13 - 15], taking the Kadomtsev-Petviashvili equation, Schwartz KdV equation and nonlinear Schrödinger equation as seed integrable models and using an extended Painlevé analysis, various higher dimensional Painlevé integrable models especially in $(3+1)$-dimensions have been obtained. Using the extended Painlevé analysis, we can also use some kinds of high dimensional Painlevé integrable conformal invariant models to solve the real high dimensional physical models [16].

Usually one can use some suitable limit procedures to get simple theories from a complicated theory. For example, most of quantum theories will be reduced to a classical one by ignoring the Planck constant $\hbar$, and a relativistic theory will be simplified to a cor- 
responding nonrelativistic one by assuming infinite light velocity. Inversely, deforming a simple theory to a complicated one is quite difficult. Fortunately, in some special cases it is possible to get significant complicated theories by deforming a simple theory. For instance, the classical Yang-Baxter equation may be evolved into the quantum Yang-Baxter equation. Some critical phenomenon theory treated by conformal field theory, where the mass is zero, may be deformed to a theory for the non-zero mass case [17]. And some special types of solutions of some simple nonlinear Klein-Gordon field equations like the single sine-Gordon and $\phi^{4}$ fields can be deformed to those of the complicated nonlinear Klein-Gordon field equations like the double sine-Gordon, $\phi^{6}, \phi^{5}$ and $\phi^{3}+\phi^{4}$ models [18 - 21].

In nonlinear physics one can obtain various lower dimensional integrable models from higher dimensional ones via some types of different approaches. In this paper we try to deform some lower dimensional simple integrable models to some higher dimensional ones especially in (3+1)-dimensions.

In Sect. 2 of this paper we discuss the general aspect of a noninvertible deformation approach and propose a special Miura type deformation relation. In Sect. 3, starting from a (0+1)-dimensional Riccati equation and the Miura type deformation relation, we obtain the general $1+1$ dimensional sinh-Gordon (SHG) and Mikhailov-Dodd-Bullough (MDB) equation. In Sect. 4 we discuss a deformation of the heat conduction equation. The Painlevé integrability of the obtained high dimensional model is proved in Sect. 5. In Sect. 6, we point out that the same result can also be obtained from the deformation of a nonintegrable nonlocal heat conduction equation by introducing the kernels of the transformation operator. Some special types of localized solutions, like multi-plane and multi-camber soliton solutions, multi-dromion solutions and multiple ring soliton solutions are listed and plotted in Sect. 7. The last section is a short summary and discussions.

\section{General Deformation Relation}

$$
\text { Let } \begin{aligned}
\phi & \equiv \phi\left(x_{1}, x_{2}, \ldots, x_{n}, y_{1}, y_{2}, \ldots, y_{m}, t\right) \text { and } \\
\phi_{t} & =K(\phi),
\end{aligned}
$$

where $K(\phi) \equiv K\left(\phi, \phi_{y_{1}}, \phi_{y_{2}}, \ldots, \phi_{y_{m}}\right)$ is a function of $\phi$ and its derivatives with respect to $y_{j}$ but not $x_{i}$ dependent explicitly, be a lower dimensional integr- able model, then a general deformation relation

$$
\begin{gathered}
B\left(x_{i}, y_{j}, t, \phi, v, \phi_{x_{i}}, \phi_{y_{j}}, v_{x_{i}}, v_{y_{j}}, \phi_{x_{i} x_{j}}, \phi_{y_{i} y_{j}},\right. \\
\left.v_{x_{i} x_{j}}, v_{y_{i} y_{j}}, \ldots\right) \equiv B(\phi, v)=0, \text { or, } \phi=\phi(v)
\end{gathered}
$$

will change the original model equation (2) to a new one. When saying the model (2) is "lower" dimensional one means (2) is not $x_{i}$-dependent explicitly. In the deformation relation ( 3$), B(\phi, v)=0$ may be $x_{i}$ dependent explicitly. The new equation can be obtained in the following way. Firstly, differentiating the transformation relation (3) with respect to time $t$ and then replacing $\phi_{t}$ by $K(\phi)$ because of (2), the final result has the form

$$
\begin{gathered}
v_{t}=\left.\left(B_{v}^{\prime}\right)^{-1} B_{\phi}^{\prime} K(\phi)\right|_{\phi=\phi(v)}+J_{1}(v) \equiv J(v), \\
\left(B_{v}^{\prime} J_{1}(v)=0\right),
\end{gathered}
$$

where the partial linearized operators $B_{v}^{\prime}$, and $B_{\phi}^{\prime}$ are defined as

$$
\begin{aligned}
& B_{v}^{\prime} f=\frac{\mathrm{d}}{\mathrm{d} \epsilon} B(\phi, v+\epsilon f)_{\epsilon=0}, \\
& B_{\phi}^{\prime} f=\frac{\mathrm{d}}{\mathrm{d} \epsilon} B(\phi+\epsilon f, v)_{\epsilon=0} .
\end{aligned}
$$

$J_{1}(v)$ is the kernel function of the operator $B_{v}^{\prime}$ and $B_{v}^{\prime}\left(B_{v}^{\prime}\right)^{-1}=1$. The inverse deformation relation with respect to (3) and (4) is

$$
\phi_{t}=K(\phi)+K_{1}(\phi) \equiv M(\phi),\left(B_{\phi}^{\prime} K_{1}(\phi)=0\right),
$$

where $K(\phi)$ is the kernel of the operator $B_{\phi}^{\prime}$.

From (2), (4) and (4'), one can see that the deformation relation (3) is only invertible if the kernels $J_{1}(v)$ and $K_{1}(\phi)$ are taken to be zero. In general, the deformation relation (3) can not preserve the integrability because of the appearance of the transformation kernels. On the other hand, because of the appearance of the kernels, one may obtain some new types of integrable models from some simple ones.

It is worth to emphasize that if there exist some more independent variables in the deformation relation (3) which do not appear explicitly in the original seed equation (2), then we say we have obtained a higher dimensional model (4) by the deformation of a "lower" dimensional one.

In the next steps we may get various new integrable models from the deformation relation (3) in two different ways. 
(I) One may fix a simple source equation (2) to get many significant models by selecting various different deformation relations. In [22], we have obtained some interesting (1+1)-dimensional and (2+1)-dimensional SHG and MDB equations by applying some different deformation relations to a quite simple $(0+1)$-dimensional Riccati equation

$$
\phi_{t}=\phi^{2},
$$

which is equivalent to a linear equation $u_{t t}=0, \phi=$ $-(\ln u)_{t}$. However, we have not yet found a suitable deformation relation to get a possible $(3+1)$-dimensional integrable model from the Riccati equation (6).

(II) We may also get some new integrable models by fixing the deformation relation (3) and selecting different source equations.

In this paper, we try to obtain some new integrable models (especially in $(3+1)$-dimensions) by using the second approach. More concretely, we will fix the deformation relation (3) as

$$
\frac{\phi_{x x x}}{\phi_{x}}-\frac{a-1}{a} \frac{\phi_{x x}^{2}}{\phi_{x}^{2}}-v_{x x}-\frac{1}{a} v_{x}^{2} \equiv B(\phi, v)=0 .
$$

It is well known that the Miura transformation

$$
u=-A v_{x x}-\frac{A}{a} v_{x}^{2}
$$

changes the solutions of the potential modified $\mathrm{KdV}$ (MKdV) equation $\left(v_{t}+v_{x x x}-\frac{2}{a^{2}} v_{x}^{3}=0\right)$ to those of the $\mathrm{KdV}$ equation $\left(u_{t}+\frac{6}{a A} u u_{x}+u_{x x x}=0\right)$. On the other hand, the relation

$$
u=-A \frac{\phi_{x x x}}{\phi_{x}}+A \frac{a-1}{a} \frac{\phi_{x x}^{2}}{\phi_{x}^{2}}
$$

changes the solutions of the Schwartz KdV equation,

$$
\frac{\phi_{t}}{\phi_{x}}+\{\phi ; x\}=0,\{\phi ; x\} \equiv \frac{\phi_{x x x}}{\phi_{x}}-\frac{3}{2} \frac{\phi_{x x}^{2}}{\phi_{x}^{2}},
$$

to those of the $\mathrm{KdV}$ equation for $a= \pm 2$. Canceling the function $u$ in (8) and (9), we get the deformation relation (7). Actually, the Miura transformation (8) is linked with not only the $\mathrm{KdV}$ and the MKdV equations but also many other significant physical models. For instance, both the Sawada-Kortera equation and the Kaup-Kupershmidt equation are linked with the Fordy-Gibbon equation by the Miura transformation (8) for suitable selections of constants $A$ and $a$ [23].
To deform the model (2) to a new one by using the Miura transformation related deformation approach, we differentiate (7) once with respect to the time $t$. The resulting equation reads

$$
e^{-\frac{2}{a} v}\left(e^{\frac{2}{a} v} v_{x t}\right)_{x}=\phi_{x}^{-\frac{2}{a}}\left(\phi_{x}^{\frac{2}{a}}\left(\phi_{x}^{-1} \phi_{x t}\right)_{x}\right)_{x},
$$

i.e.

$$
\begin{array}{r}
v_{x t}=e^{-\frac{2}{a} v}\left(\int^{x} e^{\frac{2}{a} v} \phi_{x_{1}}^{-\frac{2}{a}}\left(\phi_{x_{1}}^{\frac{2}{a}}\left(\phi_{x_{1}}^{-1} K_{x_{1}}(\phi)\right)_{x_{1}}\right)_{x_{1}} \mathrm{~d} x_{1}\right. \\
\left.+c_{0}\right)\left.\right|_{\phi=\phi(v)},
\end{array}
$$

where $c_{0} \equiv c_{0}(t, y, z, \ldots)$ is an arbitrary $x$ independent function. From (7), one can easily express the function $\phi=\phi(v)$ explicitly:

$$
\phi=-\int^{x} e^{v} u^{a} \mathrm{~d} x_{1}+c_{3}, u \equiv c_{1} \int^{x} e^{\frac{2 v}{a}} \mathrm{~d} x^{\prime}+c_{2},
$$

where $c_{1}, c_{2}$, and $c_{3}$ are all $x$-independent arbitrary functions. Using (12) and (13), we can obtain a corresponding deformed equation, whence a source equation (2) is given. According to (13), (11) can also be written as

$$
\left.K_{x}(\phi)\right|_{\phi=\phi(v)}=-e^{v} u^{a}\left(v_{t}+a u_{t} u^{-1}\right) .
$$

\section{Sinh-Gordon Equation Obtained from the Riccati Equation}

The simplest example can be obtained by selecting the source equation as the $(0+1)$-dimensional Riccati equation

$$
\phi_{t}=f_{2} \phi^{2}+f_{1} \phi+f_{0},
$$

where $f_{0}, f_{1}, f_{2}$ may be arbitrary functions of $t$. After considering that $\phi$ is not only a function of $t$ but also a function of $x$, substituting (15) into (11) yields

$$
e^{-\frac{2}{a} v}\left(e^{\frac{2}{a} v} v_{x t}\right)_{x}=\frac{2 a f_{2}}{2+a} \phi_{x x}
$$

with $\phi$ being given by (13). If $c_{1}$ of (13) is taken to be zero, (16) becomes

$$
v_{x t}=\frac{-2 a^{2} f_{2} c_{2}^{a}}{(2+a)^{2}} e^{v}+c_{0} e^{-2 v / a} .
$$


S.-y. Lou et al. · Higher Dimensional Integrable Models

For $c_{0}=0,(17)$ becomes the known Liouville field equation. For $c_{0} \neq 0$, (17) can be changed to

$w_{x \tau}=e^{w}-e^{-2 w / a}, \tau=-\int^{t}\left(\frac{2 a^{2} f_{2} c_{2}^{a} c_{0}^{a / 2}}{(a+2)^{2}}\right)^{\frac{2}{2+a}}$,

$w=v+\frac{a}{2+a} \ln \frac{a^{2} f_{2} c_{2}^{a}}{c_{0}(a+2)^{2}}$.

For $a=2$ and $a=1,(18)$ is just the (1+1)-dimensional SHG equation, and the MDB equation respectively.

It is known that for $a \neq 1,2,(18)$ is not an integrable model though it is obtained from the deformation of the trivial C-integrable Riccati equation (2). The reason is that the $c_{0} e^{-2 v / a}$ term is the kernel term of the transformation (7). In [22] we have pointed out that the general equation (16) with (13) for $a=1$ or $a=2$ is integrable. In other words, the transformation (7) preserves the integrability for the Riccati equation (15) if either the transformation kernel is taken to be zero or the transformation is restricted as $a=1$ and $a=2$.

\section{High Dimensional Nonlinear Models from the Deformation of the Heat Conduction Equation}

From (18) we know that (a) even if the source model is quite simple (which is equivalent to a linear ordinary differential equation), we can still obtain some interesting integrable nonlinear models; (b) though the source model, is an integrable model the result equation may also be nonintegrable. According to the above fact we try to obtain some high dimensional integrable models from the linear heat conduction equation

$$
\phi_{t}=\Delta \phi \equiv \sum_{k=1}^{n} \phi_{y_{k}: y_{k}}
$$

by using the deformation relation (7) with $a=2$.

Substituting (19) and $a=2$ into (11) we have

$$
v_{t}-\Delta v-\nabla v \nabla v+2\left(\ln c_{2}\right)_{t}=0, \nabla v \nabla v \equiv \sum_{k=1}^{n} v_{y_{k}}^{2}
$$

for $c_{1}=0$ and

$u^{2}\left(u_{x} u_{x t}-u_{x} \Delta u_{x}+2 \nabla u_{x} \cdot \nabla u_{x}-\left(\ln c_{1}\right)_{t} u_{x}^{2}\right)-$

$$
\begin{aligned}
& -2 u u_{x}\left(u_{x}\left(u_{t}+c_{2 t}-\Delta u\right)+2 \nabla u \cdot \nabla u_{x}\right) \\
& +2 u_{x}^{2} \nabla u \cdot \nabla u=0
\end{aligned}
$$

for $c_{1} \neq 0$. Equation (20) is a symmetrical extension of the (1+1)-dimensional potential Burgers equation $v_{t}=v_{y y}+v_{y}^{2}$ to $(n+1)$-dimensions. The integrability of the high dimensional potential Burgers equation (20) is trivial because the transformation $v=\ln (f)$ will transform it back to a linear equation (which is equivalent to the original source equation (19)). The integrability of the model equation (21) is more significant for us because we can not change the model back to the original source equation. This fact is caused by the noninvertibility of the deformation relation (7). Actually, it is known that the usual Miura transformation (8) is also noninvertible if $y_{k} \neq x$ for all $k$. It is interesting that the dimension of (21) is higher than that of the original source equation (19). More precisely, the implicit variables of (19) have become explicit ones in (21). In two special cases, $\left\{n=2, y_{1}=y, y_{2}=z\right\}$ and $\left\{n=3, y_{1}=x, y_{2}=y, y_{3}=z\right\},(21)$ describes two nonequivalent $(3+1)$-dimensional models.

\section{Painlevé Integrability of the High Dimensional Equation (21)}

Now we are interested in whether the obtained Eq. (21) is integrable or not. To clarify the integrability of a given partial differential equation (PDE), the standard Weiss, Tabor, and Carnevale (WTC) Painlevé analysis is one of the most effective methods. According to the WTC approach, if all solutions of a PDE are single valued about an arbitrary movable singular manifold, then the model possesses the Painlevé property. Many kinds of other integrable properties are linked with the Painlevé property [24]. We say a model is Painlevé integrable if it possesses the Painlevé property.

To check the Painlevé property of (21), we may expand the field $u$ as

$$
u=\sum_{j=0}^{\infty} u_{j} f^{j+\alpha},
$$

where $\alpha$ is a negative integer. Because (21) is a third order PDE, it possesses the Painlevé property only if there exists a primary branch with three arbitrary 
functions among $\left\{f, u_{j}, j=0,1,2, \ldots\right\}$. Substituting $u \sim u_{0} f^{\alpha}$ into (21) and using the leading order analysis, we find that

$\alpha=-1$, with $u_{0}$ being an arbitrary function (23)

is an only possible selection of $\alpha$ and $u_{0}$. Substituting (22) with (23) into (21) leads to the recursion relation of the expansion functions $u_{j}$ :

$$
\begin{aligned}
& j(j+1)(j-1) u_{j} \\
& \quad=F_{j}\left(u_{0}, u_{1}, u_{2}, \ldots, u_{j-1}, f_{x}, f_{y_{k}}, \ldots\right) \equiv F_{j},
\end{aligned}
$$

where $F_{j}$ is a complicated function of $u_{0}, u_{1}, u_{2}, \ldots, u_{j-1}$ and the derivatives of the manifold $f$. The left hand side of (24) shows us that the resonance occurs at $j=-1,0$, and 1 . The resonance at $j=-1$ is related to the arbitrariness of the singularity manifold $f$. The arbitrariness of $u_{0}$ shown by (23) means that the resonance condition at $j=0$ is satisfied identically. The detail calculation shows us also that the resonance condition at $j=1, F_{1}=0$, is satisfied identically. In other words, three arbitrary functions, $f, u_{0}$ and $u_{1}$ can be introduced in the general single valued expansion (22) for the third order PDE (21). So the model (21) is Painlevé integrable.

We should point out that to get the higher dimensional potential Riccati equation (20) from the heat conduction equation (19) one may use the transformation (7) for arbitrary $a$ and take the transformation kernel as zero. However, when the kernel is taken as non-zero, the detailed calculation shows us that the transformation (7) for the heat conduction equation (19) preserves the integrability only for $a=2$.

\section{A Heat Conduction Equation with a Nonlinear Nonlocal Term}

From the above discussion we know that (21) is completely nonequivalent to the original linear heat conduction equation. The linear heat conduction equation (19) is only equivalent to the potential Burgers equation (20) under the transformation relation (7) when the kernel of the operator $B_{v}^{\prime}$ is taken as zero. It is necessary to point out that the final equations (20) and (21) can also be obtained by using the same deformation relation (7) from a nonlinear non-local heat conduction equation

$\phi_{t}=\Delta \phi+C_{1}+C_{2} \phi+C_{3} \int^{x} \phi_{x_{1}} \mathrm{~d} x_{1} \int^{x_{1}} \phi_{x_{2}}^{-1} \mathrm{~d} x_{2}$,

where the last three terms with arbitrary $x$-independent functions $C_{1}, C_{2}$ and $C_{3}$ are the kernels of the transformation operator $B_{\phi}^{\prime}$. In other words, the last three terms do not offer any contribution to the result when we use the deformation relation (7). Though the transformation (7) changes (25) to the same Painlevé integrable (20) and (21), one can prove that (25) is not Painlevé integrable for $C_{3} \neq 0$.

\section{Special Solutions of the Model Equations}

Usually it is very difficult to find out some special solutions of a high dimensional nonlinear model. Fortunately, by means of the computer algebras, say Maple and Mathematica, we can prove that (21) for $c_{2}=$ constant has a solution in the form

$$
u=\frac{u_{1}}{\left.1+u_{1} \sum_{j=1}^{J} A_{j} \exp \left(k_{j}\left(x-x_{0 j}\right)+f_{j}\right)\right)},
$$

where $A_{j}, k_{j}, x_{0 j}$ are arbitrary constants , $u_{1} \equiv$ $u_{1}\left(y_{1}, y_{2}, \ldots, y_{n}, t\right)$ is an arbitrary $x$-independent arbitrary function while $f_{j} \equiv f_{j}\left(y_{1}, y_{2}, \ldots, y_{n}, t\right), j=$ $1,2, \ldots, J$ are arbitrary solutions of the overdetermined equations

$$
\begin{aligned}
& f_{j t}+\nabla f_{j} \cdot \nabla f_{j}-\Delta f_{j}-c_{1 t}+k_{j}^{2}=0, j=1,2, \ldots, n \\
& \left(k_{i}-k_{j}\right)^{2}+\left(\nabla f_{i}\right)^{2}+\left(\nabla f_{j}\right)^{2}-2 \nabla f_{i} \cdot \nabla f_{j}=0, i \neq j
\end{aligned}
$$

for one of the $y_{k}$ is equal to $x$, say $y_{1}=x$ and

$$
\begin{gathered}
f_{j t}+\nabla f_{j} \cdot \nabla f_{j}-\Delta f_{j}-c_{1 t}=0, j=1,2, \ldots, n \\
\left(\nabla f_{i}\right)^{2}+\left(\nabla f_{j}\right)^{2}-2 \nabla f_{i} \cdot \nabla f_{j}=0, i \neq j
\end{gathered}
$$

for $y_{k} \neq x, k=1,2, \ldots, n$.

It is clear that (30) has a special solution $f_{i}=f_{j} \equiv$ $f$. In the special case, $y_{k} \neq x, \forall k=1,2, \ldots, n$ and $f_{i}=f_{j} \equiv f,(26)$ becomes

$$
u=\frac{u_{1}}{1+u_{1} e^{f} \sum_{j=1}^{J}\left(A_{j} \exp \left(k_{j}\left(x-x_{0 j}\right)\right)\right)}
$$


with

$$
f_{t}+\nabla f \cdot \nabla f-\Delta f-c_{1 t}=0 .
$$

A simple special solution of (32) has the form

$$
\begin{aligned}
f=c_{1}( & t)-\ln \left\{\sum_{i, j=1}^{n} a_{i j} y_{i} y_{j}+2 t \sum_{i=1}^{n} a_{i i}\right. \\
& +\sum_{i=1}^{M}\left[\exp \left(\sum_{j=1}^{n}\left(k_{j i} y_{j}+k_{j i}^{2} t\right)+y_{0 i}\right)\right. \\
& +B_{i} \cos \left(\sum_{j=1}^{n}\left(b_{j i} y_{j}\right)\right) \exp \left(-\sum_{j=1}^{n} b_{j i}^{2} t\right) \\
& \left.\left.+C_{i} \sin \left(\sum_{j=1}^{n}\left(c_{j i} y_{j}\right)\right) \exp \left(-\sum_{j=1}^{n} c_{j i}^{2} t\right)\right]\right\}
\end{aligned}
$$

where $k_{j i}, a_{i j}, b_{j i}, c_{j i}, B_{i}, C_{i}$ and $y_{0 i}$ are arbitrary constants.

Because of the arbitrariness introduced by $u_{1}$ and $f_{j}$, the solution (26) may have a quite rich structure. In this paper we list and plot only some special interesting examples of (31) with (33):

(i) Multiple "plane" and "camber" solitons. If we take the arbitrary function $u_{1}$ as multiplane and/or multi-camber solitons in space-time $\left\{y_{1}, y_{2}, \ldots, y_{n}, t\right\}$ then (27) presents multiplane and/or multi-camber solitons in space-time $\left\{x, y_{1}, y_{2}, \ldots, y_{n}, t\right\}$. Usually, "plane" and "camber" solitons in $2+1$ dimensions are really straight and curved line solitons [22]. Figure 1a shows a four straight-line soliton solution in $(2+1)$ dimensions with $n=1, y_{1}=y, f$ is given by (33) and

$u_{1}=1, m=M=3, A_{1}=A_{3}=1, A_{2}=2$,

$k_{1}=-1, k_{2}=2, k_{3}=5, k_{11}=2, k_{12}=3, k_{13}=-2$,

$c_{1}(t)=0, x_{01}=0, x_{02}=-1, x_{03}=1$,

$B_{i}=C_{i}=a_{i j}=y_{0 i}=0$,

at time $t=0$ for the field $u$, and Fig. $1 \mathrm{~b}$ is a plot of the four straight-line soliton solution with the same condition as Fig. 1a for the potential $u_{y} \equiv u y$. Figure 1c is a plot of another type of line soliton solution in $2+1$ dimensions for the field $u$ with $n=1, y_{1}=y, f$ given by (33) with

$u_{1}=1, m=M=N=2, A_{1}=1, A_{2}=2$,

$k_{1}=-1, k_{2}=2, k_{11}=2, k_{12}=3, c_{1}(t)=0$,

$x_{01}=0, x_{02}=-1, C_{i}=a_{i j}=0, B_{1}=3, B_{2}=1$,

$b_{11}=y_{0 i}=0, b_{12}=1$.

(ii) Multiple dromion solutions. The multiple straight and curved line solitons shown by Fig. 1a, $\mathrm{b}$, and $\mathrm{c}$ are not localized in all directions. It is known that in $2+1$ dimensions, some types of solutions localized in all directions, dromions, may be driven by some straight and curved line soliton solutions for real physical fields or some types of potentials [22, 25 29]. Selecting the function $u_{1}$ of (31) appropriately, we may obtain some types of multi-dromion solutions in high dimensions. Here we write and plot down two types of point-like dromion solutions for the (21) with $c_{2}=$ const.

The first type of multi-dromion solutions may be constructed by selecting

$$
u_{1}=\sum_{j=1}^{J} \prod_{i=1}^{n} \operatorname{sech}\left(D_{i j} y_{i}-v_{i j} t+y 0_{i j}\right),
$$

where $D_{i j}, v_{i j}, y 0_{i j}, i=1,2, \ldots, n, j=1,2, \ldots, J$ are arbitrary constants.

The $2+1$ dimensional two dromion solution for the field $u$ shown by Fig. 2 has the same condition as Fig. 1c but with

$$
u_{1}=\operatorname{sech}\left(y-v_{11} t+15\right)+\operatorname{sech}\left(2 y-v_{12} t+5\right)
$$

at time $t=0$.

If we select $u_{1}$ as

$$
u_{1}=\sum_{j=1}^{J} \operatorname{sech}\left(\sum_{i=1}^{n}\left(d_{i j} y_{i}-v_{i j} t-y 0_{i j}\right)^{2}\right),
$$

where $d_{i j}, v_{i j}$ and $y 0_{i j}$ are arbitrary constants, then we may obtain a second type of high dimensional multi-dromion solutions for some suitable potentials if all the constants $d_{i j}$ are not equal to zero.

Figure 3 is an equi-potential plot of a single (3+1)dimensional dromion solution for the potential $u x \equiv$ $-u_{x}$ with the condition 

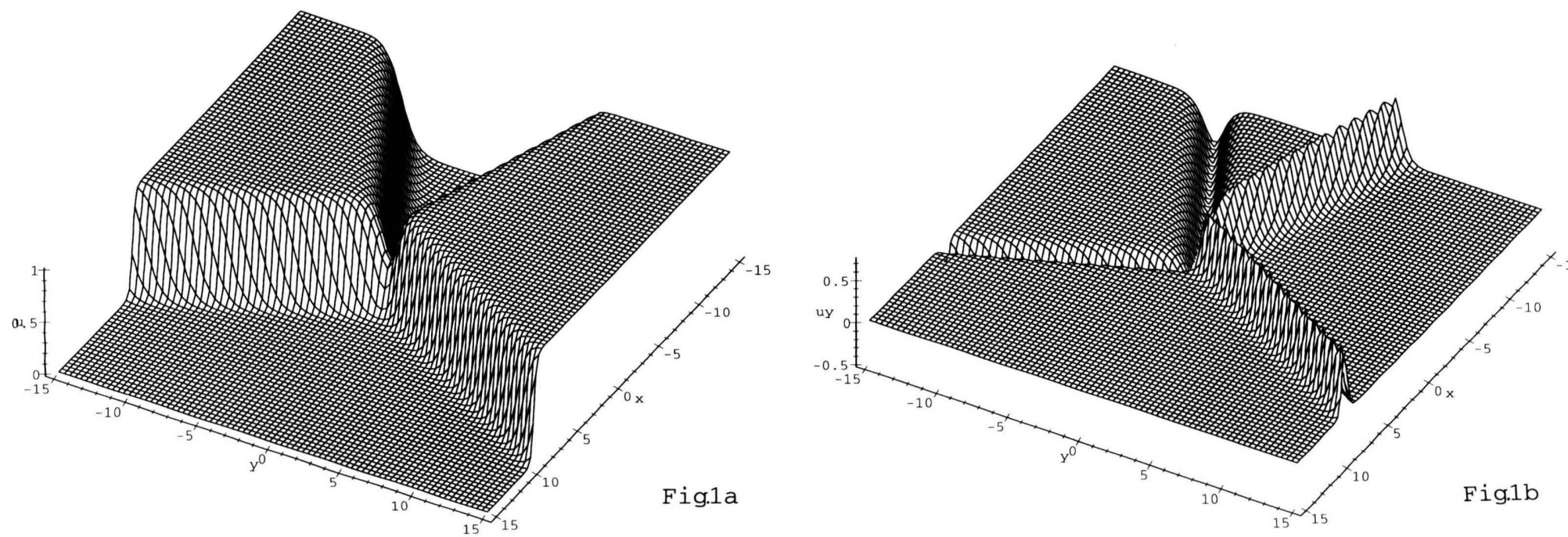

Fig. 1. Three types of multiple line soliton solutions. (a) A four straight-line soliton solution (31) in $2+1$ dimensions at time $t=0$ for the field $u$ with conditions (34) and $n=1, y_{1}=y$, while $f$ is given by (33). (b) A plot of the four straight-line soliton solution with the same condition as Fig. 1a for the potential $u_{y} \equiv u y$. (c) A plot of another type of line soliton solution in $2+1$ dimensions for the field $u$ with $n=1, y_{1}=y . f$ is given by (33) with the conditions (35) at time $t=0$.
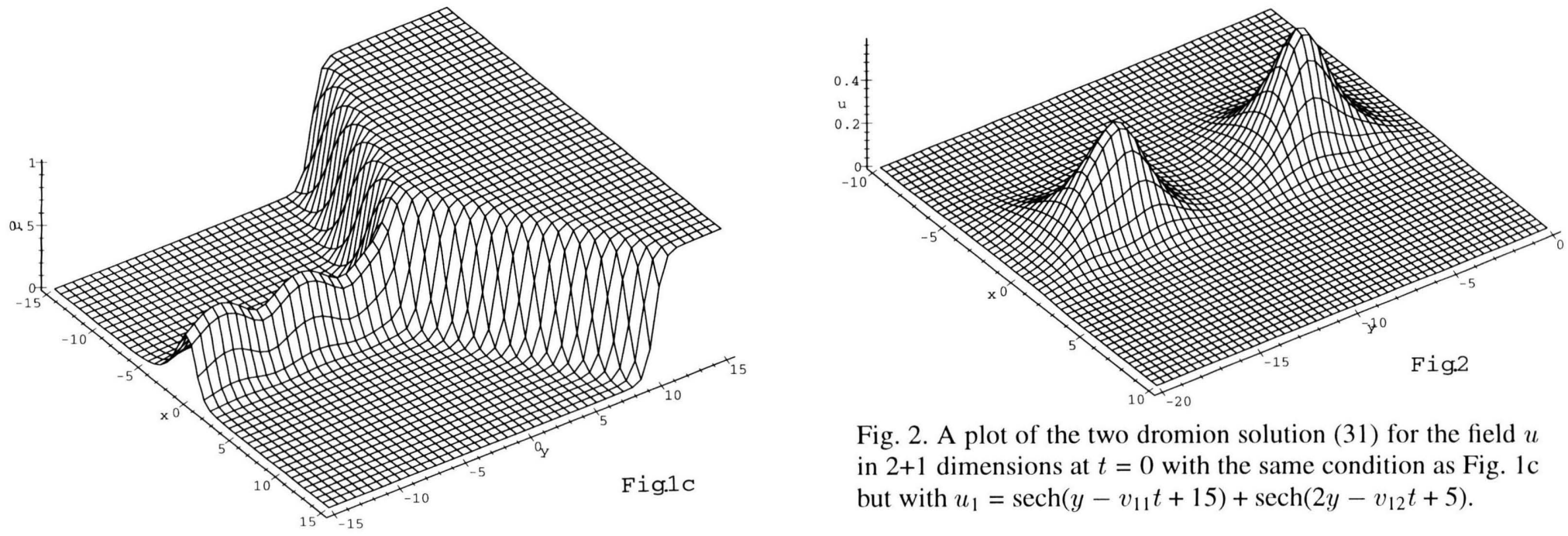

Fig. 2. A plot of the two dromion solution (31) for the field $u$ in $2+1$ dimensions at $t=0$ with the same condition as Fig. 1c but with $u_{1}=\operatorname{sech}\left(y-v_{11} t+15\right)+\operatorname{sech}\left(2 y-v_{12} t+5\right)$. 


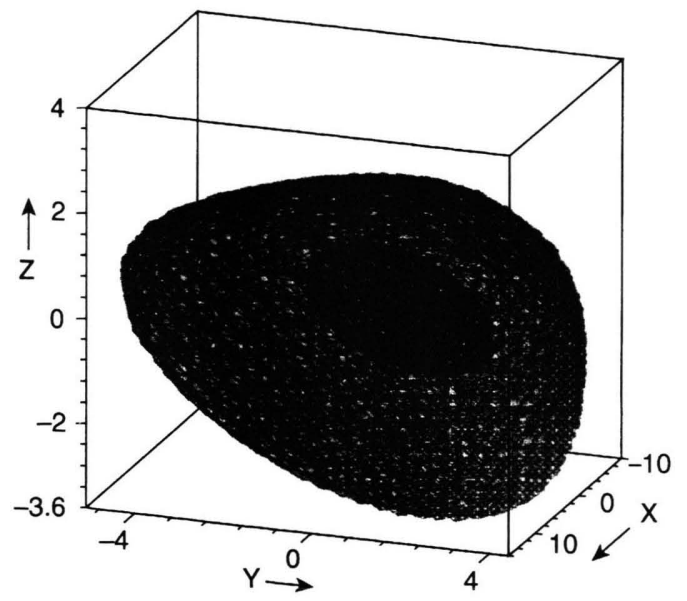

Fig. 3. An equi-potential plot of a single $(3+1)$-dimensional dromion solution for the potential $u x \equiv-u_{x}$ with the condition (39) at time $t=0$. The inner small equi-potential shell is related to $u x=0.1$ and the outside large equipotential shell is related to $u x=0.0001$.

$m=J=1, n=2, y_{1}=y, y_{2}=z$,

$B_{i}=C_{i}=a_{i j}=0, A_{1}=1, k_{1}=1, k_{11}=2, k_{12}=1$,

$c_{1}(t)=0, x_{01}=0, y 0_{11}=y_{0 i}=0$,

at time $t=0$. The inner small equi-potential shell of Fig. 3 is related to $u x=0.1$ and the outside large equi-potential shell is related to $u x=0.0001$. That is to say, as $r \equiv \sqrt{x^{2}+y^{2}+z^{2}}$ increase from $\sim 5$ to $\sim 10$, the potential $u x$ decays rapidly from 0.1 to 0.0001 and the potential $u x$ is localized in a small 3-dimensional space $\{x, y, z\}$.

(iii) Multiple ring soliton solutions. For some types of well know $(2+1)$-dimensional integrable models, like the $2+1$ dimensional sine-Gordon equation [30], Davey-Stewartson equation, NizhnikNovikov-Veselov equation and the Boiti-LeonMatina-Penpinelli equation [25], there may be some types of the ring soliton solutions (finite only at some closed curved) [31]. In 3+1 dimensions, the similar ring soliton can be driven by a ghost cylinder soliton and a ghost plane (or a camber) soliton, and the ring dromion solution is located at the cross section (a closed curve line in 3 dimensions) [29]. If we select $u_{1}$ in (31) as

$u_{1}=\sum_{j=1}^{J} \operatorname{sech}\left(\sum_{i=1}^{n}\left(d_{i j} y_{i}-v_{i j} t-y 0_{i j}\right)^{2}-r 0_{i j}^{2}\right)$, where $d_{i j}, v_{i j}, y 0_{i j}$ and $r 0_{i j}$ are arbitrary constants, then we may obtain a special type of high dimensional multiple ring soliton solutions for some suitable potentials. Actually, the second type of multi-dromion solution (31) with (38) is a special case of the multiple ring soliton solutions when all the radii of the ring solitons tend to zero.

Figure $4 \mathrm{a}$ is an equi-potential plot of a single $(3+1)$ dimensional ring soliton solution at time $t=0$ for the potential $u x \equiv-u_{x}=0.0005$ with the condition (39) and

$$
r 0_{11}=5 \text {. }
$$

Figure $4 \mathrm{~b}$ is a plot of the plane $x-2 y-z=0$ and the cylinder $y^{2}+z^{2}-25=0$ related to the ring soliton solution shown by Fig. 4a, and the ring soliton of Fig. $4 \mathrm{a}$ is located at the cross closed curve of the plane and the cylinder shown by Figure $4 \mathrm{~b}$.

\section{Summary and Discussion}

In this paper, after selecting a suitable noninvertible Miura type transformation, we have obtained some higher dimensional Painlevé integrable models from some simple lower dimensional integrable models. The (1+1)-dimensional sinh-Gordon equation can be obtained from a (0+1)-dimensional Riccati equation. A Painlevé integrable model in any space dimensions is obtained from a linear heat conduction equation and the model possesses two special $(3+1)$-dimensional nonequivalent reductions.

If we restrict the transformation kernels to zero, the transformation is invertible and the resulting equations can be transformed back to the original ones. However, if the transformation kernels are not taken as zero, the transformation is noninvertible. Three types of effects may be caused by the noninvertibility of the transformation. (I) A noninvertible transformation may destroy the integrability, say, using the transformation (7) with $a \neq 1,2$ to a simple integrable Riccati equation (15) leads to the nonintegrable model (17). (II) A suitable selection of a noninvertible transformation will lead to some new nontrivial integrable models from some trivial ones. (III). A noninvertible transformation may rule out the nonintegrability if the nonintegrable terms are just the kernels of the transformation operator, say, the transformation (7) changes the non-Painlevé integrable model (25) to the Painlevé integrable ones (20) and (21). 


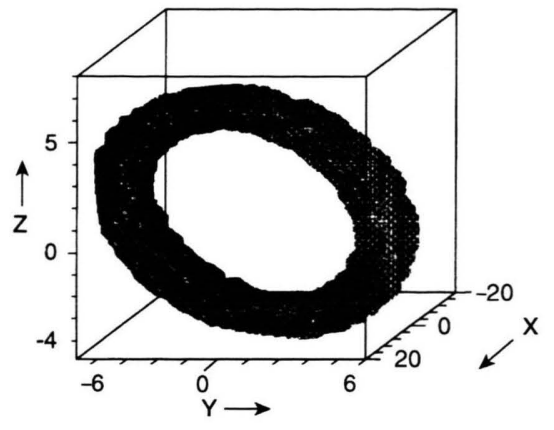

Fig. 4. (a) An equi-potential plot of a single $(3+1)$-dimensional ring soliton solution at time $t=0$ for the potential $u x \equiv$ $-u_{x}=0.0005$ with the condition (39) and $r 0_{11}=5$. (b) The plan $x-2 y-z=0$ and the cylinder $y^{2}+z^{2}-25=0$ related to the ring soliton solution shown by Fig. 4a.

A special type of exact explicit solutions of the obtained high dimensional Painlevé integrable model has been obtained also. From the special solution we see that there may be some quite rich structures for the high dimensional localized solutions. For instance, by selecting the arbitrary function $u_{1}$ appearing in the solution appropriately, we can obtain the multiple line solitons, dromion solutions and ring soliton solutions.

Because the $(1+1)$ - and $(2+1)$-dimensional integrable models and their soliton excitations have been applied widely in many fields of physics, chemistry, biology etc., one hopes that the integrable theory may also be developed well in real (3+1)-dimensional physical space-time. However, there is little progress in the study of the high dimensional integrable theory

[1] L. Dolan, Nucl. Phys. B489, 245 (1997); J. Distler and A. Hanany, Nucl. Phys. B496, 75 (1997); J. Ellis, N. E. Maoromatos, and D. V. Nanopoulos, Int. J. Mod. Phys. A12, 2639 (1997).

[2] I. Loutsenko and D. Roubtsov, Phys. Rev. Lett. 78, 3011 (1997); M. W. Coffey, Phys. Rev. B54, 1279 (1996); R. Siddhartham, B. S. Shastry, Phys. Rev. B55, 12196 (1997).

[3] M. Tajiri and H. Maesono, Phys. Rev. E55, 3351 (1997).

[4] G. C. Das, Phys. of Plasmas 4, 2095 (1997).

[5] M. Gedalin, T. C. Scott, and Y. B. Band, Phys. Rev. Lett. 78, 448 (1997); N. Ne and D. Drummond, Phys. because of the difficulty to find out high dimensional integrable models. Now we have established a systematic method to find some nontrivial integrable models from some trivial ones. More about the method and the properties of the obtained resulting equation (21), especially in $(3+1)$-dimensional reductions, are worthy of further study.

\section{Acknowledgement}

The work was supported by the National Science Foundation for young Scientists of China and the National Natural Science Foundation of China. We thank Drs. Y.-j. Zhu and H.-y. Ruan for their helpful discussions.

Rev. Lett. 78, 4311 (1997); T. Georges, Optics Lett. 22, 679 (1997).

[6] D. David, N. Kamran, D. Levi, and P. Winternitz, J. Math. Phys. 27, 1225 (1986); B. Champagne and P. Winternitz, J. Math. Phys. 29, 1 (1988); K. M. Tamizhmani and B. Gramaticos, J. Math. Phys. 32, 2635 (1991); S.-y. Lou and X.-b. Hu, J. Phys. A: Math. Gen. 27, L207 (1994); S-y Lou, Phys. Rev. Lett. 71, 4099 (1993).

[7] S.-y. Lou, J. Yu, and J. Lin, J. Phys. A: Math. Gen. 28, L191 (1995); S.-y. Lou, J. Lin, and J. Yu, Phys. Lett. A201, 47 (1995), J. Lin, S.-y. Lou and K.-l. Wang, Z. Naturforsch. 55a, 589 (2000). 
[8] S.-y. Lou, Sci. China, Series A, 34, 1317 (1997).

[9] F. Calogero and A. Degasperis, Nuovo Cimento 31B2, 201 (1976); 39B1, 54 (1977); O. I. Bogoyovlenskii, Usp. Mat. Nauk. 45, 17 (1990); Y.-s. Li and Y.-j. Zhang, J. Math. Phys. A: Math. Gen. 26, 7487 (1993).

[10] S.-y. Lou, Commun. Theor. Phys. 28, 41 (1997).

[11] S.-y. Lou and X.-b. Hu, J. Math. Phys. 38, 6401 (1997)

[12] S.-y. Lou, J. Math. Phys. 39, 2112 (1998).

[13] S.-y. Lou, Phys. Rev. Lett. 80, 5027 (1998).

[14] L.-1. Chen and S.-y. Lou, Z. Naturforsch. 53a, 689 (1998).

[15] S.-y. Lou, Sci. China, Series A, 42, 537 (1999).

[16] H.-y. Ruan, S.-y. Lou, and Y.-x. Chen, J. Phys. A: Math. Gen. 32, 2719 (1999).

[17] B. M. McCoy and J. H. H. Perk, Nucl. Phys. B 285, 279 (1987)

[18] S.-y. Lou and G.-j. Ni, J. Math. Phys. 30, 1614 (1989); Phys. Lett. A140, 33 (1989).

[19] S.-y. Lou, G.-x. Huang, and G.-j. Ni, Phys. Lett. A146, 45 (1990); S.-y. Lou, G.-j. Ni, and G.-x. Huang, Commun. Theor. Phys. 17, 67 (1992).
[20] S.-y. Lou and W.-z. Chen, Phys. Lett. A156, 260 (1991).

[21] S.-y. Lou, J. Phys. A: Math. Gen. 32, 4521 (1999); J.-s. Yang and S.-y. Lou, Z. Naturforsch. 54a, 195 (1999).

[22] S.-y. Lou, J. Phys. A: Math. Gen. 30, 7259 (1997).

[23] A. P. Fordy and J. Gibbons, Phys. Lett. A 75, 325 (1980); S.-y. Lou, J. Math. Phys. 35, 2336 (1994).

[24] J. Weiss, M. Tabor, and G. Carnevale, J. Math. Phys. 24, 522 (1983); A. Ramani, B. Gramaticos and T. Bountis, Phys. Rep. 180, 159 (1989).

[25] M. Boiti, J. J. P. Leon, L. Martina and F. Penpinelli, Phys. Lett. 132A, 432 (1988).

[26] A. S. Fokas and P. M. Santini, Phys. Rev. Lett. 63, 1329 (1989); Physia D, 44, 99 (1990).

[27] J. Hietarinta, Phys. Lett. 149A, 133 (1990).

[28] R. Radha and M. Lakshmanan J. Phys. A: Math. Gen. 35, 4746 (1994); Phys. Lett. 197A, 7 (1995).

[29] S.-y. Lou, J. Phys. A: Math. Gen. 29, 5989 (1996); Commun. Theor. Phys. 26, 487 (1996).

[30] S.-y. Lou, J. Math. Phys. 41, 6509 (2000).

[31] S.-y. Lou, Phys. Lett. A (2000), in press. 